\title{
Energy Conserving Norms for the Solution of Hyperbolic Systems of Partial Differential Equations
}

\author{
By Max D. Gunzburger* and Robert J. Plemmons**
}

\begin{abstract}
The problem of finding an energy conserving norm for the solution of the hyperbolic system of partial differential equations $\partial u / \partial t=A \partial u / \partial x$, subject to boundary conditions, is reduced to the problem of characterizing those matrices appearing in the boundary conditions which satisfy two specific matrix equations. Necessary and sufficient conditions on the coefficient matrix $A$ and the matrices appearing in boundary conditions are derived for an energy conserving norm to exist. Thus, these conditions serve as tests on a given system which determine whether or not the solution will have its energy conserved in some norm. In addition, some examples of specific systems and boundary conditions are provided.
\end{abstract}

I. Motivation. Consider the hyperbolic system of partial differential equations

$$
\frac{\partial u}{\partial t}=A \frac{\partial u}{\partial x} \text { for } t>0 \text { and } 0<x<1
$$

where $A$ is a real constant square $n \times n$ matrix. The hyperbolicity of the system (1.1) implies there exists a nonsingular real (constant) matrix $T$ which diagonalizes $A$, i.e.

$$
T A T^{-1}=\Lambda=\operatorname{diag}\left\{\lambda_{1}, \ldots, \lambda_{n}\right\},
$$

where the $\lambda_{j}$ 's, which are the eigenvalues of $A$, are real. Then the system (1.1) can be transformed into the diagonal system

$$
\frac{\partial w}{\partial t}=\Lambda \frac{\partial w}{\partial x} \text { for } t>0 \text { and } 0<x<1,
$$

where $w=T u$. Without any loss of generality we may order the eigenvalues $\lambda_{j}$ in a nonincreasing sequence, i.e. $\lambda_{1} \geqslant \cdots \geqslant \lambda_{n}$. Suppose that $p$ of these eigenvalues are positive and $q$ are negative and that $p+q=n$ (we, therefore, exclude the possibility of vanishing eigenvalues); then, we may perform the partitions

$$
\Lambda=\left(\begin{array}{cc}
\Lambda_{1} & 0 \\
0 & -\Lambda_{2}
\end{array}\right) \quad \text { and } \quad w=\left(\begin{array}{c}
w_{1} \\
w_{2}
\end{array}\right)
$$

Received August 1, 1977.

$A M S$ (MOS) subject classifications (1970). Primary 35L50, 35L65; Secondary 15A21, $65 \mathrm{~F} 35$.

* Research supported in part under NASA Contract \#NAS1-14101 while the author was in residence at ICASE, NASA Langley Research Center.

** Research supported in part by the U. S. Army Research Office under grant no. DAAG29-77-G-0166. 
where $\Lambda_{1}$ and $\Lambda_{2}$ are positive $p \times p$ and $q \times q$ diagonal matrices, respectively, and $w_{1}$ and $w_{2}$ are $p$ and $q$ vectors, respectively. Well-posed linear homogeneous boundary conditions for the systems (1.1) or (1.3) can always be written in the form (see Kreiss and Oliger [5])

$$
w_{2}(t, 0)=S_{2} w_{1}(t, 0) \text { and } w_{1}(t, 1)=S_{1} w_{2}(t, 1),
$$

where $S_{1}$ and $S_{2}$ are $p \times q$ and $q \times p$ matrices, respectively.

Given a symmetric positive definite matrix $E$, the $E$-energy is defined to be

$$
E(t)=\frac{1}{2} \int_{0}^{1}\left(u^{t} E u\right) d x
$$

The availability of a priori estimates for $E$ is important in the analysis of both analytical and numerical solutions of the system (1.1), e.g. see Gunzburger [3]. If $u$ is a solution of the system (1.1), then

$$
\frac{d E}{d t}=\frac{1}{2} \int_{0}^{1}\left(u_{x}^{t} A^{t} E u+u^{t} E A u_{x}\right) d x
$$

In order to make the integrand in (1.7) an exact differential, we require that $E$ symmetrizes $A$, i.e.

$$
E A=A^{t} E
$$

so that

$$
\frac{d E}{d t}=\frac{1}{2}\left[u^{t}(t, 1) E A u(t, 1)-u^{t}(t, 0) E A u(t, 0)\right] .
$$

The existence of a positive definite symmetrizer $E$ for a matrix $A$ has been established by Taussky [6] for exactly those matrices $A$ for which the system (1.1) is hyperbolic i.e., those matrices $A$ similar to a real symmetric matrix. Then combining (1.2) and (1.8) yields

$$
\left(T^{t}\right)^{-1} E T^{-1} \Lambda=\Lambda\left(T^{t}\right)^{-1} E T^{-1}
$$

so that $\left(T^{t}\right)^{-1} E T^{-1}$ commutes with a diagonal matrix. If the eigenvalues $\lambda_{j}$ of $A$ are distinct (the case of repeated eigenvalues is considered later), this implies that

$$
E=T^{t} D T
$$

where $D$ is a positive diagonal matrix. Equation (1.11) relates the symmetrizing matrix $E$ to the diagonalizing matrix $T$. Substitution of (1.11) into (1.6) yields

$$
E(t)=\frac{1}{2} \int_{0}^{1}\left(w^{t} D w\right) d x
$$

and if we partition $D$ as $\Lambda$ was in (1.4), i.e.

$$
D=\left(\begin{array}{cc}
D_{1} & 0 \\
0 & D_{2}
\end{array}\right),
$$

(1.2), (1.4), (1.5), (1.9) and (1.11) yield 


$$
\begin{aligned}
d E / d t=1 / 2\left\{w_{2}^{t}(t, 1)\right. & {\left[S_{1}^{t} D_{1} \Lambda_{1} S_{1}-D_{2} \Lambda_{2}\right] w_{2}(t, 1) } \\
+ & \left.\left.w_{1}^{t}(t, 0)\left[S_{2}^{t} D_{2} \Lambda_{2} S_{2}-D_{1} \Lambda_{1}\right] w_{1}(t, 0)\right]\right\}
\end{aligned}
$$

The purpose of this work is to determine what conditions the matrices $A, S_{1}$ and $S_{2}$ must satisfy in order that a matrix $E$, or equivalently $D$, exists such that the $E$ energy is conserved, i.e.

$$
d E / d t=0
$$

for arbitrary initial conditions, or equivalently, for arbitrary values of $w_{1}(t, 0)$ and $w_{2}(t, 1)$. Then comparison of (1.12) and (1.13) yields that in order for the $E$-energy to be conserved, we must have

$$
S_{1}^{t} D_{1} \Lambda_{1} S_{1}-D_{2} \Lambda_{2}=0 \text { and } S_{2}^{t} D_{2} \Lambda_{2} S_{2}-D_{1} \Lambda_{1}=0
$$

Clearly, these conditions are also sufficient.

We collect the main results of this section in the following lemmas:

LEMMA 1. Let $A$ have distinct real eigenvalues and have the decomposition (1.2). Then $E$ symmetrizes $A$ if and only if (1.11) holds for some positive diagonal matrix $D$.

LEMMA 2. If $E$ symmetrizes $A$ and if the E-energy is defined by (1.6), then the E-energy of the system (1.1) subject to the boundary conditions (1.5) is conserved if and only if (1.14) holds.

II. Characterizations of Coefficient and Boundary Matrices. Since $D_{1}, D_{2}, \Lambda_{1}$ and $\Lambda_{2}$ are all positive diagonal matrices, we may define the positive diagonal matrices $B_{1}^{2}$ and $B_{2}^{2}$ by

$$
B_{1}^{2}=D_{1} \Lambda_{1} \quad \text { and } \quad B_{2}^{2}=D_{2} \Lambda_{2}
$$

Substituting into (1.14) yields

$$
S_{1}^{t} B_{1}^{2} S_{1}=B_{2}^{2} \quad \text { and } \quad S_{2}^{t} B_{2}^{2} S_{2}=B_{1}^{2}
$$

as necessary and sufficient conditions for the conservation of $E$-energy.

In order to characterize $A, S_{1}$ and $S_{2}$ we shall make use of the following lemma:

Lemma 3. Let $U$ and $V$ be $p \times q$ and $q \times p$ matrices, respectively, and suppose $U^{t} F U=G$ and $V^{t} G V=F$, where $F$ and $G$ are nonsingular. Then, $p=q$ and $U$ and $V$ are nonsingular.

Proof. First, note that $G$ is $q \times q$ and $F$ is $p \times p$. Since the rank of the product of matrices is less than or equal to the rank of each factor, from $U^{t} F U=G$ it follows that rank $U=q$ and $q \leqslant p$; and from $V^{t} G V=F$ it follows that rank $V=p$ and $p \leqslant q$. Therefore $p=q$ and $U$ and $V$ are nonsingular.

We begin the characterization of $A, S_{1}$ and $S_{2}$ by:

THEOREM 1. If $S_{1}$ and $S_{2}$ satisfy (1.14), or equivalently (2.2), then they are square of the same size and nonsingular. As a result, if $A$ has distinct real eigenvalues, then necessary conditions for the conservation of the E-energy (where E symmetrizes $A)$ of the solution $u(x, t)$ to $(1.1)$ and (1.5) are: 
(1) A must have an even order $n$ and the same number of positive and negative eigenvalues; and

(2) The boundary condition matrices in (1.5) must be square of order $n / 2$ and nonsingular.

Proof. This theorem is an immediate consequence of Lemmas 1, 2, and 3, and the nonsingularity of $B_{1}^{2}$ and $B_{2}^{2}$.

Now that the necessity of $S_{1}$ and $S_{2}$ being nonsingular is established, definite conditions on $S_{1}$ and $S_{2}$ such that the $E$-energy is conserved are given in:

THEOREM 2. Let $A$ have distinct real eigenvalues. Then the E-energy (where $E$ symmetrizes $A$ ) of the solution $u(x, t)$ to (1.1) and (1.5) is conserved if and only if $S_{1}$ and $S_{2}^{-1}$ can be simultaneously positive diagonally scaled to orthogonal matrices, that is, there exist positive diagonal matrices $C_{1}$ and $C_{2}$ such that $C_{1} S_{1} C_{2}$ and $C_{1} S_{2}^{-1} C_{2}$ are orthogonal matrices.

Proof. Necessary and sufficient conditions for the conservation of $E$-energy are that (2.2) holds. Clearly, (2.2) may be written as

$$
\left(B_{1} S_{1} B_{2}^{-1}\right)^{t}\left(B_{1} S_{1} B_{2}^{-1}\right)=I \text { and }\left(B_{1} S_{2}^{-1} B_{2}^{-1}\right)^{t}\left(B_{1} S_{2}^{-1} B_{2}^{-1}\right)=I .
$$

Then the choice

$$
C_{1}=B_{1}=\left(D_{1} \Lambda_{1}\right)^{1 / 2} \text { and } C_{2}=B_{2}^{-1}=\left(D_{2} \Lambda_{2}\right)^{-1 / 2}
$$

yields that $C_{1} S_{1} C_{2}$ and $C_{1} S_{2}^{-1} C_{2}$ are orthogonal matrices.

On the other hand, suppose that positive diagonal matrices $C_{1}$ and $C_{2}$ exist such that $C_{1} S_{1} C_{2}$ and $C_{1} S_{2}^{-1} C_{2}$ are orthogonal matrices. Then the choice

$$
D_{1}=C_{1}^{2} \Lambda_{1}^{-1} \text { and } D_{2}=C_{2}^{-2} \Lambda_{2}^{-1}
$$

will yield (2.2) and, therefore, the $E$-energy will be conserved.

We summarize the above results in the following characterizations:

Corollary 2.1. Let the coefficient matrix $A$ of the hyperbolic system (1.1) have nonvanishing distinct real eigenvalues. Further, let $A$ have an equal number of positive and negative eigenvalues. Then, the E-energy of the solution $u(x, t)$ of $(1.1)$ and (1.5) is conserved if and only if the matrices $S_{1}$ and $S_{2}$ appearing in (1.5) belong to the class of nonsingular matrices for which $S_{1}$ and $S_{2}^{-1}$ can be simultaneously positive diagonally scaled to orthogonal matrices.

COROLLARY 2.2. Let the matrices $S_{1}$ and $S_{2}$ which appear in the boundary conditions (1.5) be square, nonsingular, and have the property that $S_{1}$ and $S_{2}^{-1}$ can be simultaneously positive diagonally scaled to orthogonal matrices. Then the E-energy of the solution $u(x, t)$ of (1.1) and (1.5) is conserved for any coefficient matrix $A$ with nonvanishing real eigenvalues which has an equal number of positive and negative eigenvalues.

Proofs. The first corollary is a restatement of Theorems 1 and 2. The second corollary is a consequence of (2.3) from which, given the scalings $C_{1}$ and $C_{2}$, the positive diagonal matrices $\Lambda_{1}$ and $\Lambda_{2}$ may be chosen arbitrarily due to the arbitrariness of $D_{1}$ and $D_{2}$. Then, choosing arbitrary nonsingular matrices $T$, we may, by the use of 
(1.2) and (1.4), construct any matrix $A$ having the properties noted in the corollary.

Remark. The matrix $A$ constructed by the process described in the proof of Corollary (2.2) need not have distinct eigenvalues since the positive diagonal matrices $\Lambda_{1}$ and $\Lambda_{2}$ can be arbitrarily chosen.

A final consequence of the results obtained above is the following construction:

COROllary 2.3. Given the matrices $A, S_{1}$ and $S_{2}$ with the necessary properties for the conservation of E-energy (these properties are given in the hypotheses of the preceding corollaries) and given the positive diagonal matrices $C_{1}$ and $C_{2}$ which simultaneously scale $S_{1}$ and $S_{2}^{-1}$ to orthogonal matrices; then a matrix $E$ for which the E-energy is conserved is given by

$$
E=T^{t}\left(\begin{array}{cc}
D_{1} & 0 \\
0 & D_{2}
\end{array}\right) T
$$

where $T$ is a diagonalizing matrix for $A$ which groups the positive and negative eigenvalues of $A$ together in the matrix $\Lambda$ given in (1.2) and where $D_{1}$ and $D_{2}$ are given by (2.3).

Proof. The result (2.4) is an immediate consequence of Lemma 1 and Theorem 2.

Remark. For given $A, S_{1}$ and $S_{2}$, the matrix $E$ will not be unique since the positive diagonal scalings cannot be unique and furthermore the diagonalizing matrix $T$ is not unique.

In view of Theorem 2 and its corollaries, there is interest in investigating pairs of matrices $S_{1}$ and $S_{2}$ that can be scaled as in Theorem 2. Conditions on $S_{1}$ and $S_{2}$ such that this is possible are given in the following theorem:

THEOREM 3. Let $S_{1}$ and $S_{2}$ be square nonsingular matrices. Then $C_{1} S_{1} C_{2}$ and $C_{1} S_{2}^{-1} C_{2}$ are orthogonal matrices for positive diagonal matrices $C_{1}$ and $C_{2}$ if and only if

$$
S_{1}^{-1}=C_{2}^{2} S_{1}^{t} C_{1}^{2} \text { and } S_{2}^{-1}=C_{1}^{-2} S_{2}^{t} C_{2}^{-2} \text {. }
$$

Moreover, in this case $C_{1} S_{1} S_{2} C_{1}^{-1}$ and $C_{2}^{-1} S_{2} S_{1} C_{2}$ are orthogonal matrices so that the eigenvalues of $S_{1} S_{2}$ and $S_{2} S_{1}$ are on the unit circle and

$$
\operatorname{det}\left(S_{1}\right)= \pm 1 / \operatorname{det}\left(S_{2}\right)
$$

Proof. Let $Q_{1}=C_{1} S_{1} C_{2}$ and $Q_{2}=C_{1} S_{2}^{-1} C_{2}$ be orthogonal matrices. It follows that

$$
S_{1}^{-1}=C_{2} Q_{1}^{t} C_{1}=C_{2}\left(C_{2} S_{1}^{t} C_{1}\right) C_{1}=C_{2}^{2} S_{1}^{t} C_{1}^{2}
$$

and

$$
S_{2}^{-1}=C_{1} Q_{2} C_{2}^{-1}=\left(C_{2} Q_{2}^{t} C_{1}\right)^{-1}=C_{1}^{-2} S_{2}^{t} C_{2}^{-2} \text {. }
$$

On the other hand, suppose that $(2.5)$ holds. Then, $\left(C_{1} S_{1} C_{2}\right)^{-1}=\left(C_{1} S_{1} C_{2}\right)^{t}$ and 
$\left(C_{1} S_{2}^{-1} C_{2}\right)^{-1}=\left(C_{1} S_{2}^{-1} C_{2}\right)^{t}$ so that $C_{1} S_{1} C_{2}$ and $C_{1} S_{2}^{-1} C_{2}$ are orthogonal matrices. The remainder of the proof then follows from the identities

$$
C_{1} S_{1} S_{2} C_{1}^{-1}=C_{1} S_{1} C_{2} C_{2}^{-1} S_{2} C_{1}^{-1}=Q_{1} Q_{2}^{t}
$$

and

$$
C_{2}^{-1} S_{2} S_{1} C_{2}=C_{2}^{-1} S_{2} C_{1}^{-1} C_{1} S_{1} C_{2}=Q_{2}^{t} Q_{1}
$$

Matrices $S_{1}$ and $S_{2}$ satisfying (2.5) with $C_{1}$ an arbitrary nonsingular matrix and $C_{2}=C_{1}^{t}$ have been studied and characterized by DePrima and Johnson [2] and Fan [3]. However, we have found no studies in the literature involving pairs of matrices that can be simultaneously positive diagonally scaled to orthogonal matrices. As a result, we give below a complete resolution to the $1 \times 1$ and $2 \times 2$ cases. In so doing the conservation of $E$-energy problem is solved for the cases where $A$ in (1.1) is of order 4 or less. (In most applications, such as acoustics, the matrix $A$ in (1.1) is indeed of order 4 or less.)

The case where $S_{1}$ and $S_{2}$ are $1 \times 1$ is especially simple. There, if $S_{1}=\left(s_{1}\right)$ and $S_{2}=\left(s_{2}\right)$, then $S_{1}$ and $S_{2}^{-1}$ can be simultaneously positive diagonally scaled to orthogonal matrices $Q=( \pm 1)$ if and only if

$$
s_{1} \neq 0 \text { and } s_{2}= \pm 1 / s_{1} .
$$

Note that with $s_{1}$ and $s_{2}$ satisfying (2.6), the positive diagonal matrices $C=\left(c_{1}\right)$ and $C_{2}=\left(c_{2}\right)$ must satisfy

$$
c_{1} c_{2}=1 /\left|s_{1}\right|
$$

but are otherwise arbitrary. Choosing $C_{1}$ and $C_{2}$ to satisfy (2.7), the matrix $E$ for which the $E$-energy is conserved can then be constructed by using (2.4).

The $2 \times 2$ case is settled by the following theorem:

THEOREM 4. Real $2 \times 2$ matrices $S_{1}$ and $S_{2}^{-1}$ can be simultaneously positive diagonally scaled to orthogonal matrices if and only if they have the representations

$$
\begin{gathered}
S_{1}=\left(\begin{array}{cc}
a c \cdot \cos \theta_{1} & \pm \mathrm{ad} \cdot \sin \theta_{1} \\
b c \cdot \sin \theta_{1} & \mp b d \cdot \cos \theta_{1}
\end{array}\right), \\
S_{2}=\left(\begin{array}{cc}
\frac{\cos \theta_{2}}{a c} & \pm \frac{\sin \theta_{2}}{b c} \\
\frac{\sin \theta_{2}}{a d} & \mp \frac{\cos \theta_{2}}{b d}
\end{array}\right)
\end{gathered}
$$

for some scalars $\theta_{1}, \theta_{2}$, and $a, b, c, d>0$.

Proof. It is known (see Cullen [1, p. 103]) that every $2 \times 2$ orthogonal matrix $Q$ has the form

$$
Q(\theta)=\left(\begin{array}{cc}
\cos \theta & \pm \sin \theta \\
\sin \theta & \mp \cos \theta
\end{array}\right) \text {. }
$$


Thus, for positive diagonal matrices

$$
C_{1}^{-1}=\operatorname{diag}\{a, b\} \quad \text { and } \quad C_{2}^{-1}=\operatorname{diag}\{c, d\},
$$

$S_{1}$ and $S_{2}$ have the form

$$
S_{1}=C_{1}^{-1} Q\left(\theta_{1}\right) C_{2}^{-1} \text { and } S_{2}=C_{2} Q\left(\theta_{2}\right) C_{1}
$$

for some $\theta_{1}$ and $\theta_{2}$. The representations (2.8) and (2.9) follow.

Remark. The $3 \times 3$ case can be similarly handled since every $3 \times 3$ orthogonal matrix has a representation in terms of two angles analogous to the $2 \times 2$ representation (2.10).

In the next section we present some illustrative examples. Before doing so, we briefly discuss the case of the coefficient matrix $A$ having repeated eigenvalues. Due to the ordering of the eigenvalues of $A$ preceding the partitioning (1.4), repeated eigenvalues are clustered together in $\Lambda$. Then (1.10) implies that $D$ is a block diagonal matrix. The diagonal blocks are all to be symmetric and positive definite so that $E$ is likewise symmetric and positive definite. The size and position of the blocks are determined in an obvious manner from the multiplicity of the eigenvalues of $A$. For example, if $\Lambda=\operatorname{diag}\{3,2,2,1,-1,-1,-2\}$, then $D$ is allowed to have the structure

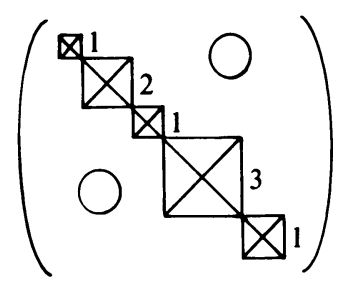

where the numbers indicate the block sizes. The lemmas, theorems and corollaries presented above are all still valid with the proviso that positive diagonal matrices are now thought of as positive definite block diagonal matrices. The validity of these results is a direct consequence of the fact that the matrices $D$ and $\Lambda$ continue to commute. We emphasize that the block diagonal structure exemplified by $(2.12)$ is the most general structure that $D$ may possess if the $E$-energy is to be conserved. On the other hand, in specific situations, the matrix $D$ may be of simpler form and still the $E$-energy would be conserved. (See the remark following Corollary 2.2.)

III. Examples. The first example, though admittedly simple, illustrates many of the points discussed in the previous section. Let

$$
A=\left(\begin{array}{ll}
\mu & 1 \\
1 & \mu
\end{array}\right) \quad \text { and } \quad u=\left(\begin{array}{l}
u_{1} \\
u_{2}
\end{array}\right)
$$

where $0<\mu<1$ and let the boundary conditions be given by

$$
u_{1}(t, 0)=u_{1}(t, 1)=0
$$


Obviously, $A$ is of even order and the eigenvalues of $A$ are given by

$$
\lambda_{1}=\mu+1>0 \text { and } \lambda_{2}=\mu-1<0 .
$$

The diagonalizing matrix for $A$ may be taken to be

$$
T=\frac{1}{\sqrt{2}}\left(\begin{array}{rr}
1 & 1 \\
1 & -1
\end{array}\right)=T^{-1}=T^{t},
$$

so that $w=T u$ and the boundary conditions (3.2) yield

$$
w_{2}(t, 0)=-w_{1}(t, 0) \text { and } w_{1}(t, 1)=-w_{2}(t, 1)
$$

Therefore, $S_{1}=S_{2}=(-1)$ so that (2.6) is satisfied. Then, according to (2.7) we may choose $C_{1}=C_{2}=(1)$. Since $\Lambda_{1}=(1+\mu)$ and $\Lambda_{2}=(1-\mu),(2.3)$ yields that

$$
D_{1}=1 /(1+\mu) \text { and } D_{2}=1 /(1-\mu) \text {. }
$$

Then, with $T$ given by (3.3), (2.4) yields that

$$
E=\frac{1}{\left(1-\mu^{2}\right)}\left(\begin{array}{rr}
1 & -\mu \\
-\mu & 1
\end{array}\right) \text {. }
$$

We now check to see if indeed $E$ satisfies all our requirements. It is obvious that $E$ is symmetric and since its eigenvalues are $1 \pm \mu, E$ is positive definite. Furthermore,

$$
E A=\left(\begin{array}{ll}
0 & 1 \\
1 & 0
\end{array}\right)
$$

so that $E$ symmetrizes $A$. Finally, the $E$-energy is conserved since from (1.9) and (3.2) we see that

$$
d E / d t=1 / 2\left\{u_{1}(t, 1) u_{2}(t, 1)-u_{1}(t, 0) u_{2}(t, 0)\right\}=0 .
$$

If the boundary conditions (3.4) are replaced by the conditions $w_{2}(t, 0)=$ $\nu w_{1}(t, 0)$ and $w_{1}(t, 1)=\sigma w_{2}(t, 1)$, then $|\nu \sigma|=1$ is necessary and sufficient for the conservation of $E$-energy. Furthermore, the scalars $D_{1}$ and $D_{2}$ must be chosen so that

$$
D_{1} \Lambda_{1}=\nu^{2} D_{2} \Lambda_{2}
$$

This simple example is of special interest since its $E$-energy can be related to a physical energy. With $A$ given by (3.1) the system (1.1) is an equivalent to Galilean transformation of the wave equation, and the $E$-energy of the solution of (1.1) is equivalent to the physical energy measured in the transformed space-time coordinates (see Gunzburger [4] for details).

Less trivial examples may be built by choosing boundary matrices of the form (2.8) and (2.9) and also choosing a matrix $\Lambda$. Then coefficient matrices $A$ may be constructed from (1.2) by choosing any nonsingular matrix $T$. Furthermore, using (2.3), (2.4) and (2.11), the $E$ matrix may be constructed. For illustration purposes, let the parameters in (2.8) and (2.9) be given by $\theta_{1}=\pi / 2, \theta_{2}=\pi / 3, a=d=1$, and $b=c=2$. Then 


$$
S_{1}=\left(\begin{array}{cc}
0 & \pm 1 \\
4 & 0
\end{array}\right) \text { and } S_{2}=\left(\begin{array}{cc}
1 / 4 & \pm \sqrt{3} / 8 \\
\sqrt{3} / 2 & \pm 1 / 4
\end{array}\right)
$$

are boundary matrices such that $S_{1}$ and $S_{2}^{-1}$ can be simultaneously positive diagonally scaled to orthogonal matrices. In fact, from (2.11) we see that if $C_{1}^{-1}=\operatorname{diag}\{1,2\}$ and $C_{2}^{-1}=\operatorname{diag}\{2,1\}$, then

$$
C_{1} S_{1} C_{2}=\left(\begin{array}{cc}
0 & \pm 1 \\
1 & 0
\end{array}\right) \text { and } C_{1} S_{2}^{-1} C_{2}=\frac{1}{2}\left(\begin{array}{cc}
1 & \sqrt{3} \\
\sqrt{3} & \mp 1
\end{array}\right)
$$

are indeed orthogonal. If $\Lambda=\operatorname{diag}\left\{\lambda_{1}, \lambda_{2},-\lambda_{3},-\lambda_{4}\right\}$, where all the $\lambda_{j}$ 's are positive, then from (2.3) we may choose

$$
D=\operatorname{diag}\left\{1 / \lambda_{1}, 1 / 4 \lambda_{2}, 4 / \lambda_{3}, 1 / \lambda_{4}\right\}
$$

Then, from (1.12), it can easily be verified that the $E$-energy is conserved.

As further illustrations we list some zero patterns of matrices $S_{1}$ and $S_{2}$ satisfying Theorem 4. Matrices

$$
S_{1}=\left(\begin{array}{cc}
s_{11} & 0 \\
0 & s_{22}
\end{array}\right), \quad S_{2}=\left(\begin{array}{cc}
t_{11} & 0 \\
0 & t_{22}
\end{array}\right)
$$

satisfy Theorem 4 if and only if $s_{11} s_{22} \neq 0$ and $t_{11}= \pm 1 / s_{11}$ and $t_{22}= \pm 1 / s_{22}$. Matrices

$$
S_{1}=\left(\begin{array}{cc}
0 & s_{12} \\
s_{21} & 0
\end{array}\right), \quad S_{2}=\left(\begin{array}{cc}
0 & t_{12} \\
t_{21} & 0
\end{array}\right)
$$

satisfy Theorem 4 if and only if $s_{12} s_{21} \neq 0$ and $t_{12}= \pm 1 / s_{21}$ and $t_{21}= \pm 1 / s_{12}$. Moreover, combining these two situations, it can be shown more generally that

$$
S_{1}=\left(\begin{array}{cc}
s_{11} & 0 \\
0 & s_{22}
\end{array}\right), \quad S_{2}=\left(\begin{array}{cc}
0 & t_{12} \\
t_{21} & 0
\end{array}\right)
$$

satisfy Theorem 4 if and only if

$$
s_{11} s_{22} t_{12} t_{21}= \pm 1
$$

For by Theorem 3, (3.5) is necessary and a simple analysis shows that if (3.5) holds then $S_{1}$ and $S_{2}$ satisfy (2.8) and (2.9), respectively, with say $\theta_{1}=k \pi, k$ even if $s_{11}>0, k$ odd if $s_{11}<0$, with $\theta_{2}=(2 k+1) \pi / 2, k$ even if $t_{21}>0, k$ odd if $t_{21}<$ 0 , and with say $a>0$ arbitrary and

$$
b=a /\left|s_{11}\right|\left|t_{21}\right|, \quad c=\left|s_{11}\right| / a, \quad d=1 / a\left|t_{12}\right| .
$$

Other pattern pairs can be characterized in a similar manner.

We conclude with the remark that if $A$ is symmetric then the $l_{2}$ energy $(u, u)$,

$$
\int_{0}^{1} u^{t} u d x
$$

is conserved if and only if $E=D=I, S_{1}^{t} \Lambda_{1} S_{1}=\Lambda_{2}$ and $S_{2}^{t} \Lambda_{2} S_{2}=\Lambda_{1}$. 
Acknowledgement. We are grateful to Professor James Ortega for his helpful comments during the work on this paper.

Department of Mathematics

The University of Tennessee

Knoxville, Tennessee 37916

Department of Computer Science and

Department of Mathematics

The University of Tennessee

Knoxville, Tennessee 37916

1. C. G. CULLEN, Matrices and Linear Transformation, Addison-Wesley, Reading, Mass., 1966.

2. C. DePRIMA AND C. JOHNSON, "The range of $A^{-1} A^{*}$ in $G L(n, C)$," Linear Algebra and Appl., v. 9, 1974, pp. 209-222.

3. KY FAN, "On strictly dissipative matrices," Linear Algebra and Appl., v. 9, 1974, pp. 223-241.

4. M. D. GUNZBURGER, "On the stability of Galerkin methods for initial boundary value problems for hyperbolic systems," Math. Comp., v. 31, 1977, pp. 661-675.

5. H. O. KREISS AND J. OLIGER, Methods for the Approximate Solution Time Dependent Problems, Global Atmospheric Research Programme Publ. Ser., no. 10, Geneva, 1973.

6. O. TAUSSKY, "Positive definite matrices and their role in the study of characteristic roots of general matrices," Advances in Math., v. 2, 1968, pp. 175-186. 